\title{
ESTUDO DO CAMPO DE VENTO DO RIO DE JANEIRO: UTILIZANDO MODELOS NUMÉRICOS ATMOSFÉRICOS NA IDENTIFICAÇÃO DAS ÁREAS ATINGIDAS PELA EMISSÃO DE FULIGEM DA CSA EM 2010
}

\author{
FARIAS, Heitor Soares - heisofa@gmail.com
}

\begin{abstract}
RESUMO
O uso de modelos na ciência é antigo e seu grande valor está na possibilidade de organizar a complexidade da realidade em um quadro simplificado, permitindo obter conclusões que possam ser reaplicadas ao mundo real. O uso de modelos para representação da atmosfera tem sido muito difundido, principalmente os modelos numéricos, que são importantes para prognosticar a formação e evolução de sistemas de escala regional, como também avaliar fatores ambientais dinâmicos e termodinâmicos em escala local. O Brazilian Regional Atmospheric Modeling System (BRAMS) é um exemplo de modelo numérico atmosférico, desenvolvido por pesquisadores brasileiros, para representar melhor o estado da atmosfera dos trópicos, que admite resolução suficiente para prognosticar fenômenos meteorológicos, proporcionando bons resultados com diferentes resoluções espaciais para uma mesma área. É possível obter alta resolução espacial em uma área de maior interesse enquanto cobre uma área muito maior com menor resolução. Devido a essas características, o BRAMS foi escolhido para ser aplicado na cidade do Rio de Janeiro que, com grande contraste no relevo, alternando planícies e maciços ao longo da costa, apresenta dificuldades para circulação do ar. Posteriormente, utilizando os resultados do BRAMS é possível alimentar outro modelo, chamado de Trajetórias Cinemáticas 3D, que permite visualizar a trajetória dos poluentes em três dimensões. Assim, utilizando um episódio de emissão de fuligem, provocado pela Companhia Siderúrgica do Atlântico (CSA), em dezembro de 2010, o objetivo deste trabalho é validar os dois modelos numéricos na cidade do Rio de Janeiro - BRAMS e Trajetórias Cinemáticas 3D -, no maior nível de resolução, verificando se são capazes de reproduzir a interferência do relevo na circulação dos ventos, e, identificar corretamente as áreas atingidas pela poluição.

Palavras chave: Poluição do ar, bacias aéreas, modelos atmosféricos, CSA, Rio de Janeiro.
\end{abstract}

STUDY OF WIND FIELD OF RIO DE JANEIRO: USING ATMOSPHERIC NUMERICAL MODELS IN IDENTIFICATION OF AREAS AFFECTED BY THE ISSUANCE OF SOOT IN 2010 CSA

\section{ABSTRACT}

The use of models in science is old and its great value lies in the possibility to organize the complexity of reality in a simplified, allowing to draw conclusions that can be reapplied to the real world. The use of models to represent the atmosphere has been very widespread, especially numerical models, which are important to predict the formation and evolution of regional systems, as well as evaluating environmental dynamic and thermodynamic factors at the local scale. The Brazilian Regional Atmospheric Modeling System (BRAMS) is an example of atmospheric numerical model, developed by Brazilian researchers, to better represent the state of the atmosphere of the tropics, which admits resolution sufficient to predict meteorological phenomena, providing good results with different spatial resolutions for a same area. It is possible to obtain high spatial resolution in an area of great interest as it covers a much larger area with a lower resolution. Due to these characteristics, the BRAMS was chosen to be applied in the city of Rio de Janeiro, who in great contrast in relief, alternating plains and massifs along the coast, presents difficulties for air circulation. Subsequently, using the results of BRAMS is possible to feed another model, called the kinematic trajectories 3D to visualize the trajectory of pollutants in three dimensions. Thus, using an episode of soot, caused by Atlantic Steel Company (CSA), in December 2010, the goal of this work is to validate the two numerical models in the city of Rio de Janeiro - BRAMS and 3D kinematic trajectories - the highest resolution, checking if they are able to reproduce the interference of relief in wind circulation, and correctly identify the areas affected by pollution.

Keywords: Air pollution, air basins, atmospheric models, CSA, Rio de Janeiro.

\section{INTRODUÇÃO}

O uso de modelos na ciência é antigo e seu grande valor está na possibilidade de organização das complexidades da realidade em um quadro simplificado, pois têm como objetivo geral a obtenção de conclusões para que sejam reaplicadas 
ao mundo real. Os modelos são aproximações altamente subjetivas por não incluírem todas as observações, "mas são valiosos por obscurecerem detalhes acidentais e por permitirem 0 aparecimento dos aspectos fundamentais da realidade" (CHORLEY E HAGGETT, 1975, p.4). Nesse sentido a modelagem numérica apresenta-se como uma ferramenta de grande importância tanto para prognosticar a formação e evolução de sistemas de mesoescala, como para diagnosticar e avaliar fatores ambientais dinâmicos e termodinâmicos associados (COTTON et al., 1982; FLATAU et al., 1989; PIELKE et al., 1992).

A modelagem numérica apresenta-se hoje como um poderoso método na pesquisa de variados fenômenos nos diversos campos do conhecimento, especialmente aqueles não reprodutíveis em condições controladas. Uma grande vantagem da modelagem numérica atmosférica é o fato de conseguir obter campos com resolução espacial e temporal muito maior do que as informações disponíveis na rede sinótica. No entanto, como a grande maioria dos modelos vem de outros países, e não representam a realidade dos países tropicais, deve ser definido um procedimento padrão para avaliar a precisão desses modelos.

A correta utilização dos modelos de transporte e difusão de poluentes na atmosfera não pode prescindir de um estudo sobre a capacidade de representarem corretamente situações reais. Sempre que possível deve-se comparar os dados simulados com os observados na área de estudo, pois modelos considerados seguros nos EUA podem não ter o mesmo comportamento quando aplicados em uma configuração particular no Brasil (FREITAS et al., 1996; FREITAS, 1999).

O Regional Atmospheric Modeling System (RAMS), desenvolvido na Universidade do Colorado (EUA), a partir de um modelo de mesoescala (PIELKE, 1974) e de um modelo de nuvens (TRIPOLI E COTTON, 1980), é um modelo de circulação atmosférica que realiza prognósticos. Ele está fundamentado na integração de equações diferenciais, conservação de momentum, massa e energia, referenciado em um sistema de coordenadas geográficas. É um modelo bastante complexo, capaz de simular o escoamento atmosférico em distintas situações sinóticas, possibilitando diferentes tipos de estudo que envolvam informações sobre previsão do tempo.

Com o objetivo de reproduzir as condições atmosféricas locais, pesquisadores brasileiros desenvolveram o modelo Brazilian Regional Atmospheric Modeling System (BRAMS), um modelo meteorológico de mesoescala baseado no RAMS. Os modelos RAMS e BRAMS são bastante semelhantes, só que o segundo foi desenvolvido com alguns ajustes para representar melhor o estado da atmosfera dos trópicos, apresentando bons resultados em relação às condições atmosféricas brasileiras.

O BRAMS é um modelo que admite resolução suficiente para prognosticar fenômenos meteorológicos de mesoescala, que variam de um a centenas de quilômetros. Essa versatilidade é possível, também, devido ao recurso de grades aninhadas que proporciona bons resultados com diferentes resoluções espaciais 
para uma mesma área, sendo possível obter alta resolução espacial em uma área de maior interesse enquanto cobre uma extensão muito maior com menor resolução. A interação entre as grades aninhadas ocorre em duplo sentido (twoway interaction), da grade de maior para a de menor resolução e vice-versa, resolvendo as equações simultaneamente (CLARK E FARLEY, 1984; CLARK E HALL, 1991).

As equações usadas no BRAMS são para uma atmosfera não hidrostática (TRIPOLI E COTTON, 1982), que permite descrever processos físicos onde a velocidade vertical é intensa. Os processos de interação entre a atmosfera e a superfície são representados numericamente, simulando a difusão turbulenta, a radiação solar, a formação e interação de nuvens, a precipitação de hidrometeoros (líquido e gelo), a convecção de cumulus, a troca de calor sensível e latente (incluindo o papel da vegetação) e o transporte de calor no solo. Para todas essas variáveis de estado da atmosfera, incluindo os ventos (u, v, w), temperatura potencial, razão de mistura e função de Exner, o modelo possui equações capazes de fazer prognósticos (FARIAS, 2012).

Assim, por suas características, é possível utilizar o modelo BRAMS para prognosticar a circulação dos ventos em pequena escala, e, posteriormente, aplicar os resultados em outro modelo, de Trajetórias Cinemáticas 3D, criado por Freitas (1999), que permite visualizar a circulação dos poluentes. Esta possibilidade foi o que mais chamou a atenção para a aplicação dos modelos na cidade do Rio de Janeiro, já que devido ao grande contraste de seu relevo, que alterna planícies e maciços que se elevam a 900 metros de altitude, em média, apresenta dificuldades para circulação do ar, e nos últimos anos tem sofrido com as emissões de fuligem e poeira metálica da Companhia Siderúrgica do Atlântico (CSA).

A CSA é uma grande usina siderúrgica de alta performance que fabricará placas de aço no bairro de Santa Cruz, na Zona Oeste do Rio de Janeiro. Como a produção da CSA é totalmente voltada para o mercado externo, a localização do empreendimento em Santa Cruz é fundamental devido a proximidade da Baía de Sepetiba, onde há condições para a implantação de um terminal portuário próprio. Há também a possibilidade de reabilitação do ramal ferroviário existente nas adjacências, além da existência de um distrito industrial consolidado (EIA, 2005). No entanto, o funcionamento do empreendimento é bastante controvertido, pois a população tem reclamado das sucessivas emissões de fuligem (duas vezes em 2010) e poeira metálica (em 2012), que têm causado problemas respiratórios aos moradores vizinhos à CSA, fato que já resultou a aplicação de três multas por parte do Instituto Estadual do Ambiente (INEA).

Desta maneira, utilizando um dos eventos de emissão de fuligem pela CSA, em dezembro de 2010, o presente trabalho tem como objetivo validar dois modelos numéricos na cidade do Rio de Janeiro - BRAMS e Trajetórias Cinemáticas 3D -, no maior nível de resolução, verificando se são capazes de reproduzir a 
interferência do relevo na circulação dos ventos, e, identificar corretamente as áreas atingidas pela poluição.

\section{MATERIAL E MÉTODOS}

Para testar os modelos, foi escolhido um evento amplamente divulgado pela mídia: as partículas em suspensão emitidas acidentalmente pela CSA que, no dia 26 de dezembro de 2010, atingiu a população do bairro de Santa Cruz, no Rio de Janeiro, nas proximidades da Avenida Brasil, por volta das 6 horas da manhã.

O modelo BRAMS foi rodado com três grades aninhadas e alimentado com as condições iniciais e de contorno gerado pelo modelo global do CPTEC. O recurso das grades aninhadas, que proporciona bons resultados com diferentes resoluções espaciais para uma mesma área, permitiu aproximar ao nível de detalhe de uma bacia aérea. A grade com maior resolução espacial, grade 3 ou fina, com dimensão de 38 pontos na direção leste-oeste e 42 na direção nortesul, com distância de $2,5 \mathrm{~km}$ entre os pontos, com o centro localizado nas coordenadas $22,95^{\circ} \mathrm{S}$ e $43,70^{\circ} \mathrm{W}$, cobriu o domínio das bacias aéreas. É importante destacar que nesse nível de resolução alcançado pela grade 3, a imagem que reproduz a velocidade e direção dos ventos, inclui, diferenciado por cores, a altimetria do relevo.

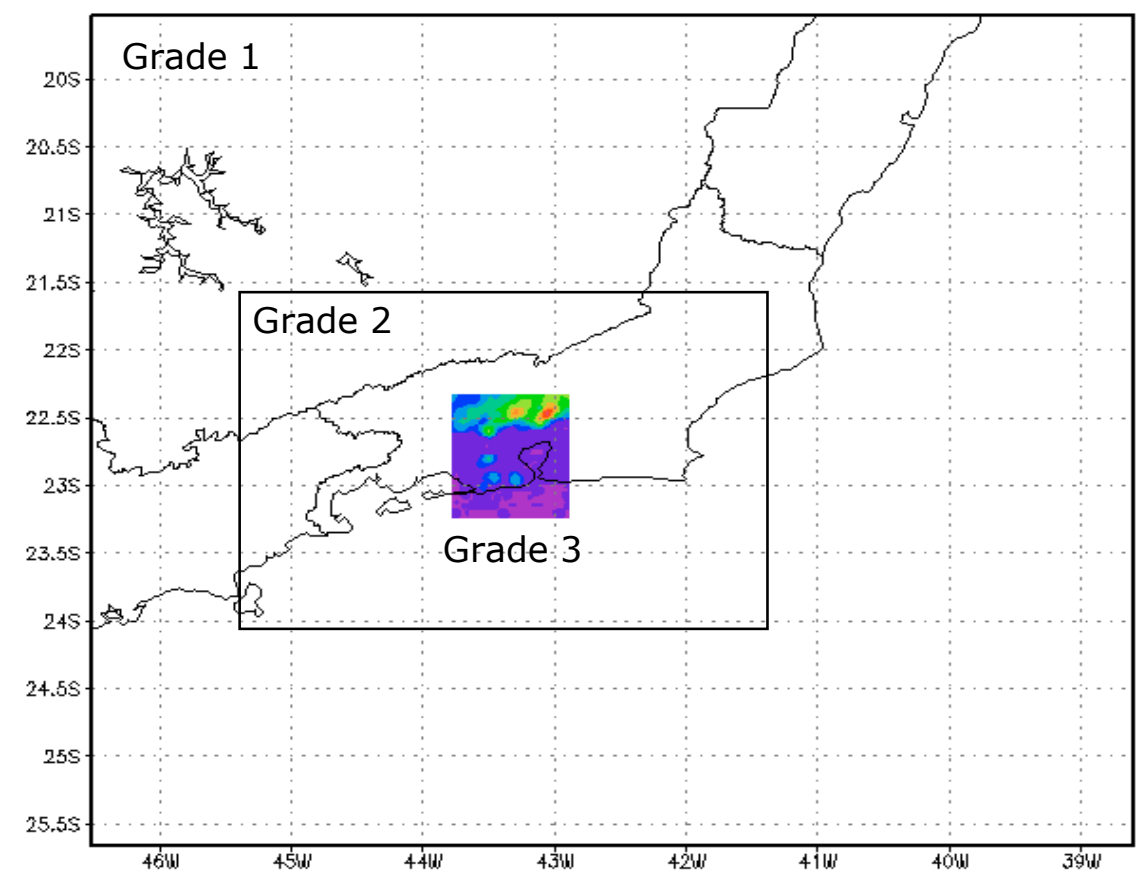

Figura 1 - Sobreposição de grades sobre o Estado do Rio de Janeiro, onde a grade 3, também chamada de fina é a de maior resolução, de $2,5 \times 2,5 \mathrm{~km}$. Fonte: Adaptado de Oliveira (2004) 
Para simular as trajetórias dos poluentes emitidos na RMRJ optou-se pelo modelo cinemático tridimensional e método não convectivo (FREITAS, 1999), alimentado com dados sobre o campo de vento gerado pelo modelo BRAMS. Como trata-se de um modelo tridimensional representado em uma superfície, a altitude de uma partícula ou poluente, ao longo da trajetória de parcelas de ar, é representada por uma escala de cores, variando do azul marinho para altitudes de até 100 metros, até o rosa para altitudes entre 1900 e 2000 metros.

As trajetórias foram simuladas a partir da altura de 70 metros, nas coordenadas 22, $9066 \mathrm{~S}$ e 43, $7404 \mathrm{~W}$, para simular a emissão de uma chaminé da indústria CSA; de hora em hora desde a noite do dia 25 de dezembro de 2010 até a manhã do dia 26 de dezembro de 2010, quando os moradores perceberam a presença de fuligem no bairro de Santa Cruz. Para visualização das trajetórias foi utilizado o software GRADS.

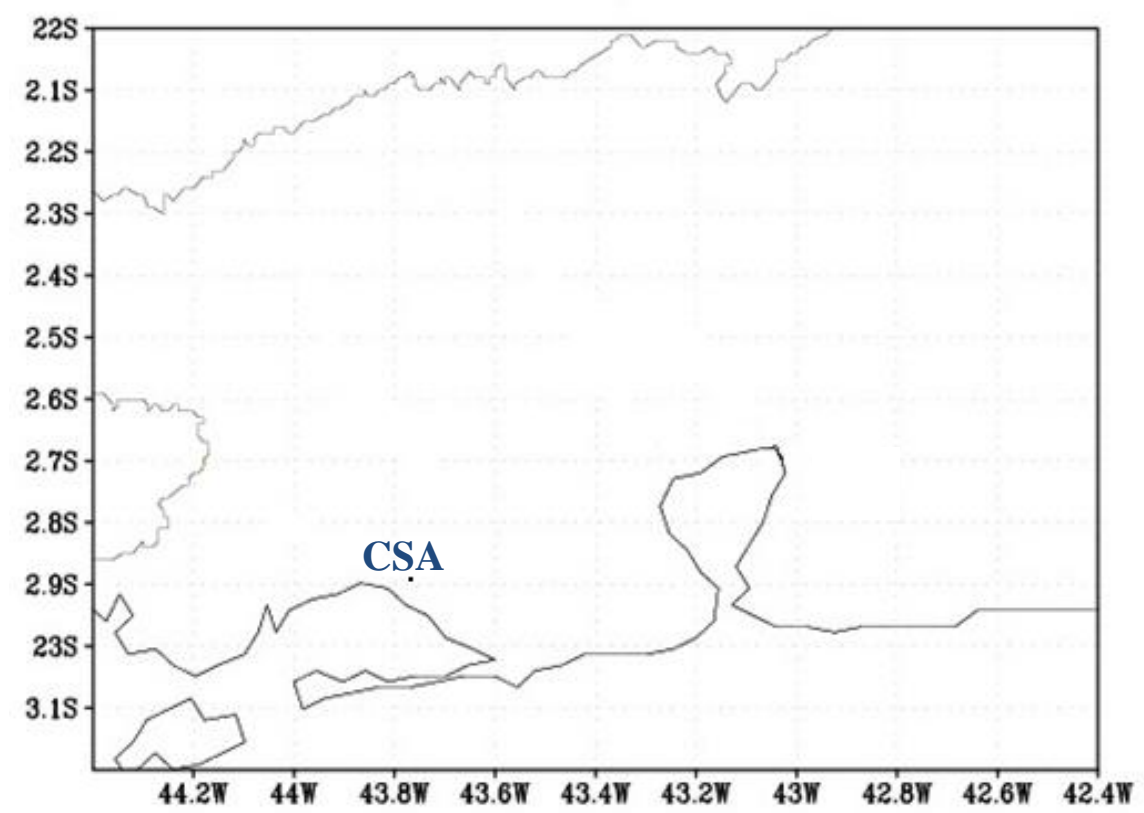

Figura 2 - Localização do ponto de emissão para simulação das trajetórias 3D a partir das indústrias CSA.

\section{RESUltados e discussão}

Antes de utilizar os modelos foi preciso averiguar as informações publicadas nos jornais. Os moradores de Santa Cruz, nas proximidades da Avenida Brasil (Figura 3), relataram que a fuligem começou a cair por volta das 6 horas da manhã, podendo ter sido emitida durante a madrugada ou mesmo na noite anterior.

Em nota a CSA informou que um defeito em um guindaste obrigou o descarte de ferro gusa. Os ventos fortes fizeram com que poeira de grafite alcançasse as comunidades vizinhas na manhã do dia 26 (O Globo, 2010). Assim, também de 
acordo com as informações passadas pela CSA, não ficou explícito que a emissão ocorreu às 6 horas da manhã, pois os ventos fortes da manhã é que levaram as partículas para as áreas vizinhas neste horário.

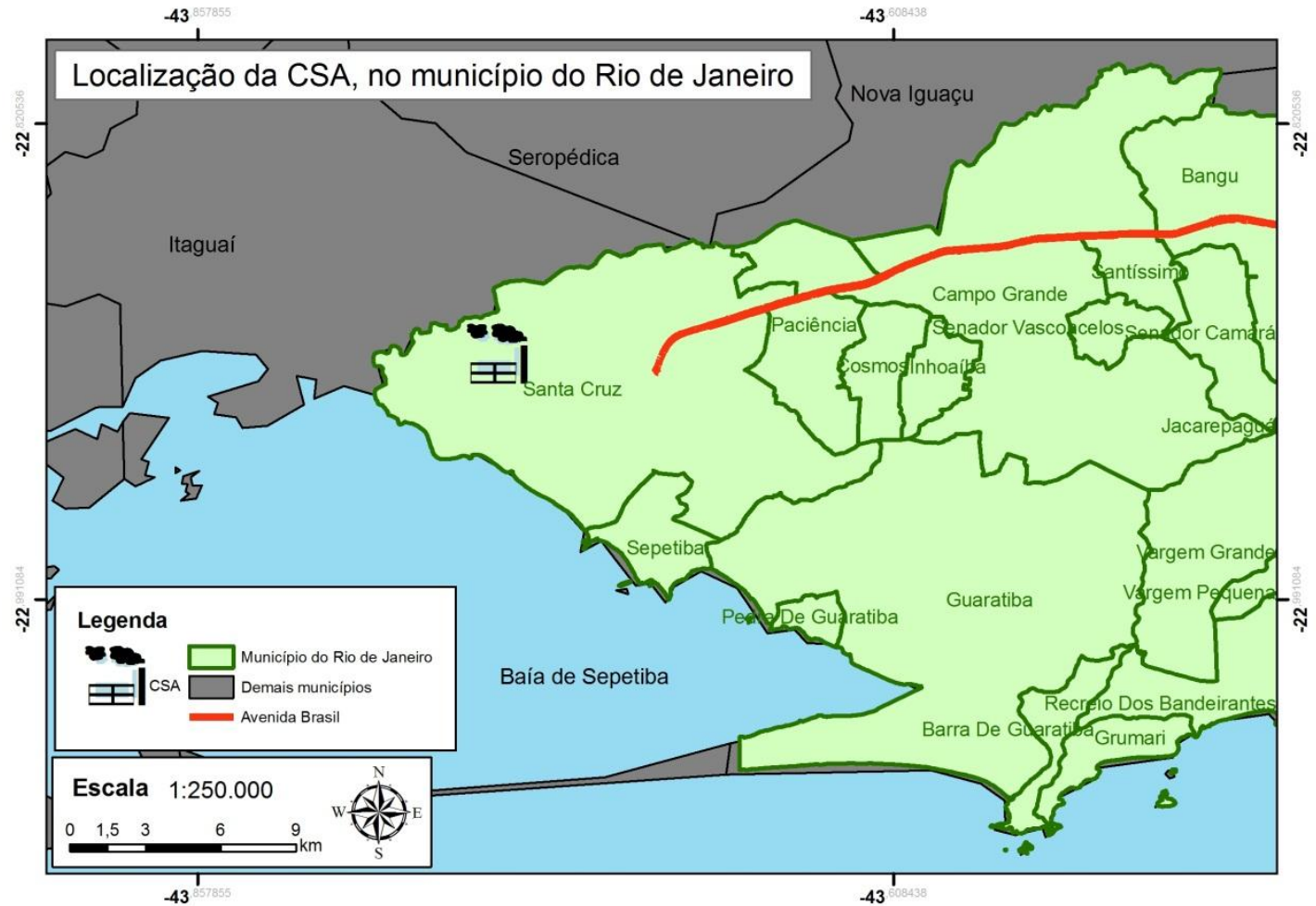

Figura 3 - Localização da CSA em relação à Avenida Brasil e aos bairros da Zona Oeste do Rio de Janeiro.

Utilizando-se a imagem de satélite (Figura 4) e a carta sinótica (Figura 5) verificou-se a presença de forte nebulosidade a noroeste do Estado do Rio Grande do Sul, e uma frente fria se aproximando do Estado de São Paulo, na madrugada do dia 25 para 26 de dezembro de 2010. Na imagem de satélite observa-se uma forte nebulosidade próxima ao Rio de Janeiro. 

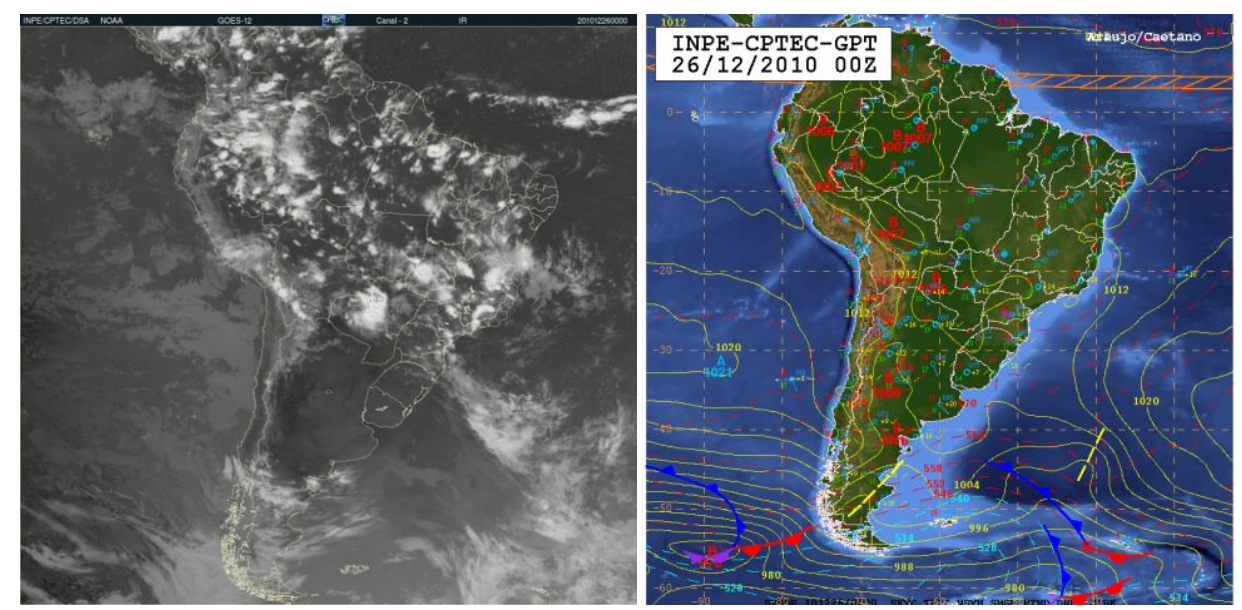

Figuras 4 e 5 - Imagem de satélite e carta de superfície do dia 26 de dezembro de 20100 UTC

A presença das frentes frias, que percorrem o litoral sul do Brasil apresentando uma trajetória de sudoeste para nordeste, que tem na retaguarda do sistema frontal a penetração do anticiclone polar migratório, favorece o escoamento de sul no Rio de Janeiro (RODRIGUES et al., 2004), em detrimento da ação do Anticiclone Subtropical do Atlântico Sul, quando são observados ventos de quadrante norte sobre o estado fluminense.

A Figura 6, simulada com o modelo BRAMS, mostra o escoamento do tipo brisa marítima atuando. O modelo considera o relevo em diferentes altitudes formando um corredor que canaliza os ventos que entram pela Baía de Sepetiba. Os ventos seguem pelos bairros da Zona Oeste, inclusive Santa Cruz, e são direcionados para a área onde localiza-se a Avenida Brasil, uma das principais vias de circulação da cidade. 


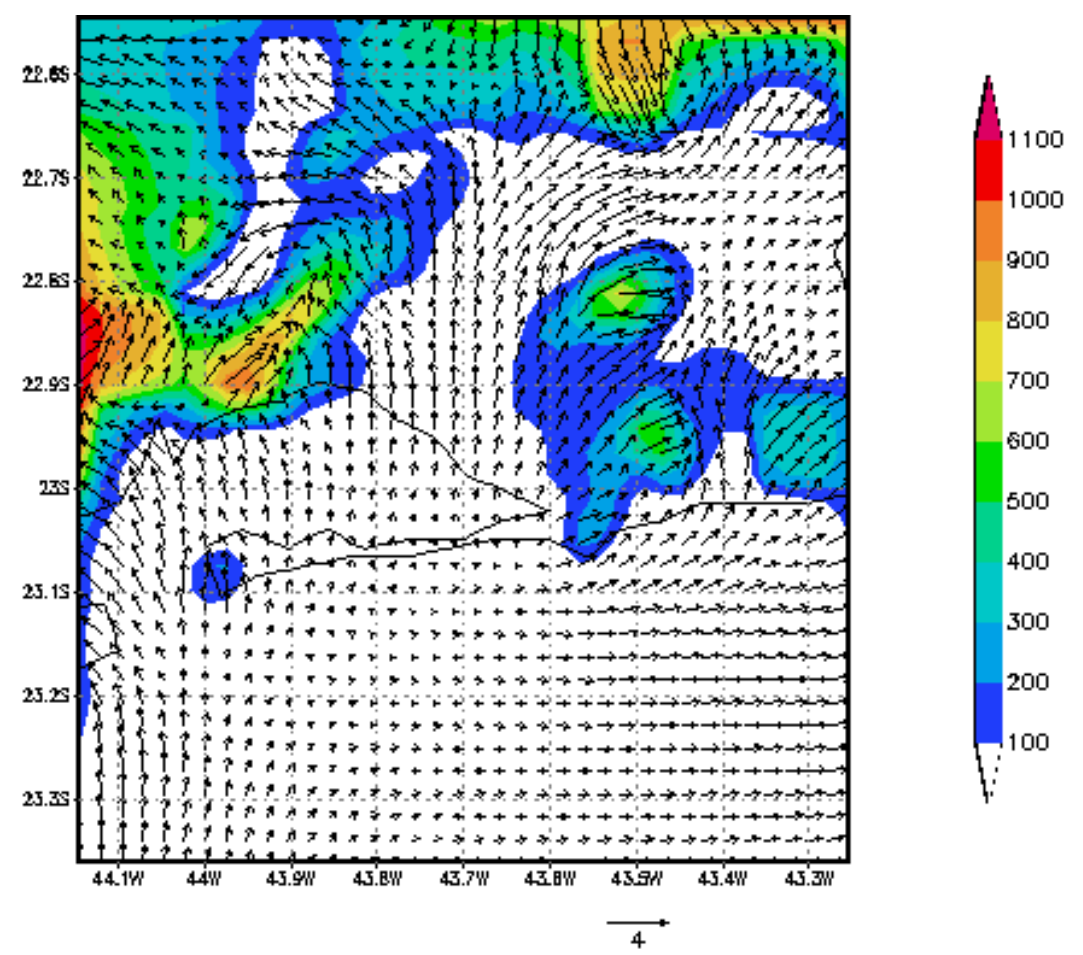

Figura 6 - Escoamento do tipo brisa em 25 de dezembro de 2010, 21 horas

A partir do campo de ventos simulados com o BRAMS foi possível calcular as trajetórias dos poluentes emitidos pela CSA no dia 25 de dezembro de 2010, às 21 horas (Figura 7). As trajetórias se deslocam em baixa altitude passando por áreas próximas da indústria a menos de 100 metros do solo, e seguem em direção à Avenida Brasil, onde estão as áreas mais atingidas, confirmando o relato dos moradores, segundo O Globo (2010). 


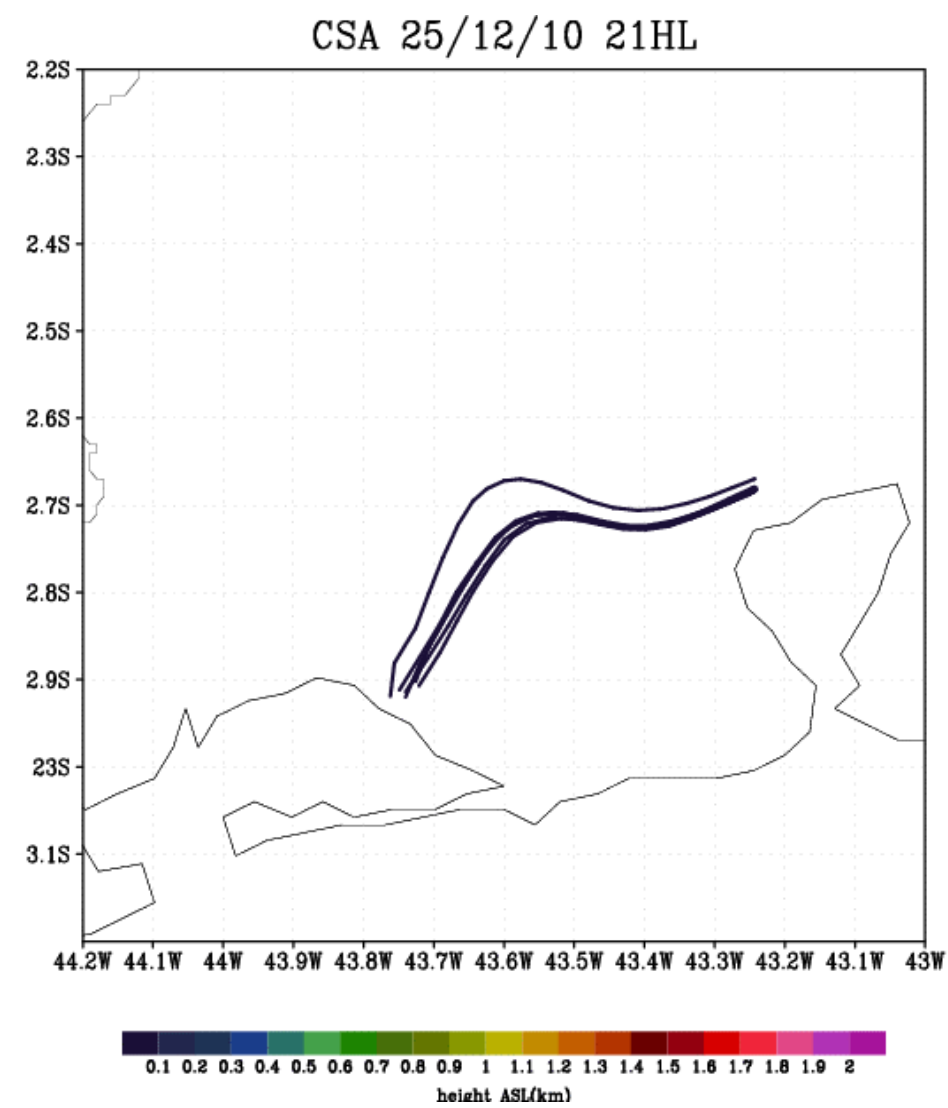

Figura 7 - Trajetórias partindo da CSA no dia 25 de dezembro de 2010, 21 horas local

\section{CONCLUSÕES}

O modelo BRAMS foi eficiente ao indicar a mudança de comportamento na circulação dos ventos, ainda que estivéssemos trabalhando com a grade 3 , de maior resolução. Foi possível observar as setas surgindo no canto inferior esquerdo da imagem, indicando que os ventos estavam chegando a partir do quadrante sul, que é uma característica provocada pela aproximação de um sistema frontal.

Utilizando os dados sobre a circulação dos ventos gerados pelo BRAMS, o modelo Trajetórias Cinemáticas 3D também mostrou-se eficiente. Embora a qualidade cartográfica da imagem seja muito fraca em detalhes, demonstra precisão e permitiu ter uma boa noção do deslocamento dos poluentes emitidos pela CSA. Observou-se que na noite do dia 25 de dezembro, por volta das 21 horas, devido às condições atmosféricas, os poluentes não ganharam altitude, deslocando-se a menos de 100 metros da superfície, em direção à Avenida Brasil. Assim, apesar de as fuligens terem sido observadas na manhã do dia 26 de dezembro, os resultados mostraram que as condições atmosféricas da noite anterior eram as mais adequadas para que o material particulado alcançasse os bairros vizinhos. Desta maneira, conclui-se que os modelos BRAMS e Trajetórias Cinemáticas 3D foram validados, mostrando-se importantes ferramentas na análise do 
comportamento da atmosfera, sendo valiosos mesmo quando utilizados em uma área de grande resolução, calculando dados para áreas influenciadas pela presença do relevo.

\section{REFERÊNCIAS}

CLARK, T. L; FARLEY, R. D. Severe downslope windslope windstorm calculation in two and three spatial dimensions using anelastic interactive grid nesting. A possible mechanism for gustiness. J. Atmos. Sci., 41, 329-350, 1984.

CLARK, T. L \& HALL, W. D. Multi-domain simulations of the time dependent Navier-Stokes equations: Benchmark error analysis of some nesting procedures. J. Comput. Phys., 92, 456-481, 1991.

CHORLEY, R. J. E HAGGETT, P. Modelos, paradigmas e a nova geografia. São Paulo. Editora da Universidade de São Paulo. pp. 1 - 19, 1975.

COTTON, W. R.; STEPHENS, M. A.; NEHRKORN, T. et al. Three-dimensional cloud/mesoscale model parameterization- 1982. J. Rech. Atmos., v. 16. p. 295-320, 1982.

EIA - ESTUDO DE IMPACTO AMBIENTAL DA USINA SIDERÚRGICA CSA. Reference: B6000/05.01. Outubro, 2005.

FARIAS, H. S. Espaços de risco à saúde humana na região metropolitana do Rio de Janeiro: um estudo das trajetórias de poluentes atmosféricos do Arco Metropolitano, CSA e COMPERJ. Tese de Doutorado. Instituto de Geociências da Universidade Federal Fluminense, Niterói. 149p, 2012.

FLATAU, P. J.; TRIPOLI, G. J.; VERLINDE, J. et al. The CSU-RAMS cloud microphysics module: general theory and code documentation Colorado State University. Atmospheric Science, Paper NP 451.88 p, 1989.

FREITAS, S. R.; LONGO, K. M.; DIAS, M. A. F. S.; ARTAXO, P. Numerical modeling of air mass trajectories from the biomass burning areas of the Amazon Basin. An. Acad. Bras. Ci., n.68. 193-206p, 1996.

FREITAS, S. R. Modelagem numérica do transporte e das emissões de gases traços e aerossóis de queimadas no cerrado e floresta tropical da América do Sul. Tese de doutorado. Instituto de Física da Universidade de São Paulo, São Paulo. 185p, 1999.

JORNAL O GLOBO on line - Disponível em http://oglobo.globo.com/rio/mat/2010/12/26/csa-recebe-30-dias-de-prazopara-dar-solucao-definitiva-para-poluicao-em-santa-cruz-923357134.asp Acessado em 27/12/2010.

OLIVEIRA, J. L. F. Análise espacial e modelagem atmosférica: contribuições ao gerenciamento da qualidade do ar da bacia aérea III da região metropolitana do Rio de Janeiro. Tese de Doutorado - COPPE Universidade Federal do Rio de Janeiro, Rio de Janeiro. 144p, 2004. 
PIELKE, R. A.; COTTON, W. R.; WALKO, R. L., et al. A comprehensive meteorological modeling system - RAMS. Meteorol. Atmos. Phys., v. 49, pp. 69-91, 1992.

RODRIGUES, M. L. G.; FRANCO, D.; SHIGETOSHI, S. Climatologia de frentes frias no litoral de Santa Catarina. Revista Brasileira de Geofísica v.22, p. 135-151, 2004.

TRIPOLI, G.J. AND COTTON, W.R. A Numerical Investigation of Several Factors Leading to the Observed Variable Intensity of Deep Convection over South Florida. J. Appl. Meteor.,19,1037-1063, 1980.

TRIPOLI, G. J.; COTTON, W.R. The Colorado University Three-Dimensional Cloud/Mesoescale Model Part I: General Theorical Framword and Sensivity Experiments. Journal of Research Atmospheric, v. 16, p. 185-220, 1982. 